\title{
Predictive Analytics of In-Service Bridge Structural Performance from SHM Data Mining Perspective: A Case Study
}

\author{
Qiwen Jin, ${ }^{1,2}$ Zheng Liu, ${ }^{3}$ Junchi Bin, ${ }^{4}$ and Weixin Ren ${ }^{5}$ \\ ${ }^{1}$ PhD. Candidate, School of Civil Engineering, Hefei University of Technology, Hefei, China \\ ${ }^{2}$ Visiting Student, School of Engineering, University of British Columbia, Kelowna, BC, Canada \\ ${ }^{3}$ Associate Prof., School of Engineering, University of British Columbia, Kelowna, BC, Canada \\ ${ }^{4}$ Master Graduate, School of Engineering, University of British Columbia, Kelowna, BC, Canada \\ ${ }^{5}$ Prof., School of Civil Engineering, Hefei University of Technology, Hefei, China \\ Correspondence should be addressed to Weixin Ren; renwx@hfut.edu.cn
}

Received 15 April 2019; Revised 13 May 2019; Accepted 28 May 2019; Published 1 July 2019

Guest Editor: Franco Concli

Copyright (c) 2019 Qiwen Jin et al. This is an open access article distributed under the Creative Commons Attribution License, which permits unrestricted use, distribution, and reproduction in any medium, provided the original work is properly cited.

\begin{abstract}
In-service bridge structural performance analysis and prediction are usually complicated and challenging because of many unknown and uncertain factors. Contrary to the traditional structural appearance inspections and load tests, structural health monitoring (SHM) can provide a perspective for online analysis, prediction, and early warning. So far, SHM has been widely used in many bridge structures, and a lot of bridge SHM data have also been collected. However, the existing studies usually focus on some independent and unsystematic analysis methods, which are hard to use widely in engineering applications to reveal the overall structural performance. This study focuses on the structural performance analysis and prediction of the highway in-service bridge. The dynamic problems in bridge SHM are pointed out firstly, followed by a detailed analysis about the characteristics of bridge SHM data. With the consideration of different characteristics, three targeted analysis methods are proposed. An urban concrete-filled steel tube (CFST) truss girder bridge (opened to traffic in 1995) is also presented, which once experienced some prominent vibration problems. The bridge SHM system is designed and stalled after several appearance inspections, load tests, and some reinforcement measures. The data mining methods proposed (distribution function, association analysis, and time-series analysis) are employed for the analysis and prediction of structural response and deterioration extent. This study can provide some references for maintenance and management and can also build a foundation for further online analysis and early warning.
\end{abstract}

\section{Introduction}

In-service bridge structural performance analysis is usually complicated because of many unknown and uncertain factors. Contrary to the traditional structural appearance inspections and load tests, the predictive analytics of inservice bridge structural performance with SHM data mining can provide a perspective for online analysis and early warning. Actually, SHM has been widely used in largespan bridges and even some medium-span bridges, e.g., the Brooklyn Bridge in New York, the Sutong Bridge in China, and the first Bosphorus Bridge in Turkey [1-3].

With the help of the SHM system, a large amount of bridge SHM data can then be collected, including dynamic response and physical response [4], which makes it possible for further structural performance analysis and prediction. However, the in-service bridge structural performance analysis was usually based on structural dynamic properties, such as the modal analysis [5]. Several methods were also provided for the structural deterioration analysis, damage detection, and assessment, such as the vibration signature analysis and the mode shape variation analysis [6-9]. Some large bridge case studies have also been carried out, such as the deflection distribution estimation of a tied-arch bridge, the tuned mass damper performance evaluation of a continuous steel box-girder bridge, and the dynamic property investigation of a cablestayed bridge $[10,11]$.

Data mining firstly appeared at the 11th International Conference on Artificial Intelligence (1989, Detroit), and then the first international conference was held in Montreal 
(1995) $[12,13]$. SHM data mining is basically a logical inference problem, part of which can also be used to handle the multi-temporal and multi-sensor data [14]. So far, data mining has been widely used to extract the information from SHM data, such as the artificial neural network and the principal component analysis [15]. Several studies have already been carried out. For example, a Bayesian network was constructed based on the domain knowledge and the spatial data. The Bayesian network aims to facilitate the integration of multiple factors and the quantification of uncertainties within a consistent system [16]. A density-based clustering technique is further optimized, aiming to identify the data density and noisy data elements from a large test data set of a steel truss bridge [17]. Based on the significant factors that caused structural damage, the Apriori and Ripper algorithms were applied for the pattern analysis. The machine learning concept was also employed for resampling and upgrading the estimation model [18]. The support vector machine (SVM) is also applied to formulate the regression models, and a squared correlation coefficient is also defined for optimizing the SVM coefficients [19]. The cluster analysis method, association analysis method, and time-series analysis method are also employed for bridge SHM data anomalies, aiming to find the rules between bridge structure and environmental parameters [20]. Several classification methods and models were also selected to classify the damage, and then predict the lateral spread displacement of concrete buildings [21, 22]. Moreover, the current challenges in SHM data are also discussed, including the characteristics of large SHM data sets and the expected processing challenges associated with these data sets [23]. In general, the current studies are usually based on some single and unsystematic data mining methods. Moreover, the analysis method and model are also hard to use widely in engineering practice to reflect the overall structural performance.

This study focuses on the structural performance analysis and prediction of an in-service bridge with the targeted SHM data mining methods. In this paper, essential dynamic problems in bridge SHM and the characteristics of bridge SHM data are pointed out, and then three targeted analysis methods are proposed. An urban concrete-filled steel tube (CFST) truss girder bridge (opened to traffic in 1995) is presented. The bridge once experienced some prominent vibration problems. The SHM system is also designed and installed, after several appearance inspections, load tests, and some reinforcement measures. Three data mining methods (distribution function, association analysis, and time-series analysis) are employed for predictive analytics of structural response and deterioration extent. The results can provide a reference for the maintenance and traffic management department, and can also build a foundation for the further online analysis and early warning.

\section{Predictive Analytic Methodology}

2.1. Dynamic Problems in Bridge SHM. The bridge structural response is essentially a structural dynamic problem. The traffic load is the external excitement $(f(x))$, the bridge structure is usually characterized by different physical and vibration parameters, and then different kinds of structural responses can be generated $(x(t))$. Their relationship is shown in Figure 1.

According to Figure 1, supposing the external traffic load and the bridge structure are known, different responses can then be calculated. This can be named the forward problem, which is very common in the design stage of a bridge so as to test whether it can meet the design requirement, while the predictive analytics of structural performance and even the external traffic load can be called the inverse problems. The former one (analysis and prediction of structural performance) is typically used in the assessment of some old or damaged bridges, which supposes the external traffic or at least the structural response is known. The latter one (analysis and prediction of traffic load) usually supposes the bridge structure and the response are known or at least the response is known. Both of them can be used for the online analysis and prediction with SHM data mining, and then provide some references for the maintenance and management department.

2.2. Characteristics of Bridge SHM Data. The bridge monitoring data collected by the SHM system, in essence, are the structural response of a bridge subjected to different traffic loads. Specifically, the structural response can be characterized by the mass data, the variation along with different traffic loads and different time periods, and the variation along with different measurement positions and different physical attributes.

Consider a simply supported girder bridge with five small T-type beams as an example. Suppose the SHM system has five strain sensors and five deflection sensors, and the measurement interval is one minute. If the storage space of a single sensor is about $4 \mathrm{MB}$ per day, it will be about $120 \mathrm{MB}$ a month and $1400 \mathrm{MB}$ a year. Thus, the different structural response of the whole bridge will be about $14000 \mathrm{MB}$. Moreover, there will be many different bridge SHM systems and even hundreds of sensors in a management center. Therefore, a huge high-performance computer will be needed to store and view the SHM data.

Additionally, the structural response usually changes along with the variation of different traffic and different time periods. With the help of a video monitoring system, the structural response can be clearly observed. For a highway bridge, there are more traffic during the daytime (mainly passenger cars, buses, and trucks) and less traffic during the nighttime (mainly trucks). The SHM data of daytime are intensive with high values, while different responses of nighttime are discrete, and a larger variation can only be observed when the truck passes. For the urban bridge, there is more traffic during the daytime (mainly the passenger cars, buses, and some trucks). On the contrary, there is almost no traffic during the nighttime. Therefore, different responses mainly concentrate on the daytime.

Moreover, most of the bridges are designed with two or even more lanes and consisted of several different small beams with a wide lateral cross section. Usually, the outside 


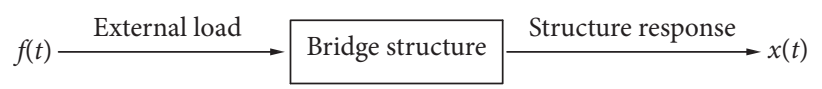

Figure 1: Diagram of the bridge structure, external load, and structural response.

lane is arranged for trucks, the inside lane is arranged for passenger cars, and the middle lane is arranged for mixture traffic. Thus, different beams will carry different traffic. The structural responses of different beams will vary greatly at any instant time, and then the structural lateral distribution performance can be reflected. So far, several bridge accidents have occurred because of the weak lateral distribution performance, such as the single beam or slab fracture.

For an intact bridge structure, different structural parameters are usually based on the structural mechanics and material mechanics with a good mapping relationship. However, there will be a different deterioration extent after a long periodical service. The mapping relationship of structural stress and displacement effect will then be gradually weakened, which can be properly analyzed and predicted with the data mining methods.

2.3. Analysis Methods. Data mining is usually based on the traditional statistical methods, aiming to discover some new meaningful associations, patterns, and trends from a large amount of data. Data mining has some significant advantages, such as convenience for enterprise users without professional statistical background [13]. Data mining now has more than ten calculation methods, such as the distribution function analysis, association analysis, and timeseries analysis.

2.3.1. Distribution Function Analysis. The traditional statistical method is usually based on the overall samples or part of samples, aiming to describe the distribution status of data sets. The traditional statistical analysis is usually along with the frequency distribution histogram or empirical distribution function, such as the multi-modal distribution.

Consider the multi-modal probability distribution as an example. Based on some approximated conditions, the multi-modal probability distribution can be described as the weighted sum of two normal distributions, three normal distributions, and even multi-modal normal distribution. Figure 2 shows the diagram of a bi-modal distribution density function curve. It can also be described as the weighted sum of two normal distribution density function curves. The multi-modal distribution formula is shown as [24]

$$
F_{X}(x)=\sum_{i=1}^{n} p_{i} \phi\left(\frac{x-\mu_{i}}{\sigma_{i}}\right)
$$

where $p_{i}, \mu_{i}$, and $\sigma_{i}$ are the probability, mean, and standard deviation of the $i$ th normal distribution, respectively. $\sum_{i=1}^{n} p_{i}=1, \sigma=\sqrt{\sum_{i=1}^{n} p_{i} \sigma_{i}^{2}+\sum_{i=1}^{n} p_{i}\left(\mu-\mu_{i}\right)}$, and $\mu \sum_{i=1}^{n} p_{i} \mu_{i}=1$.

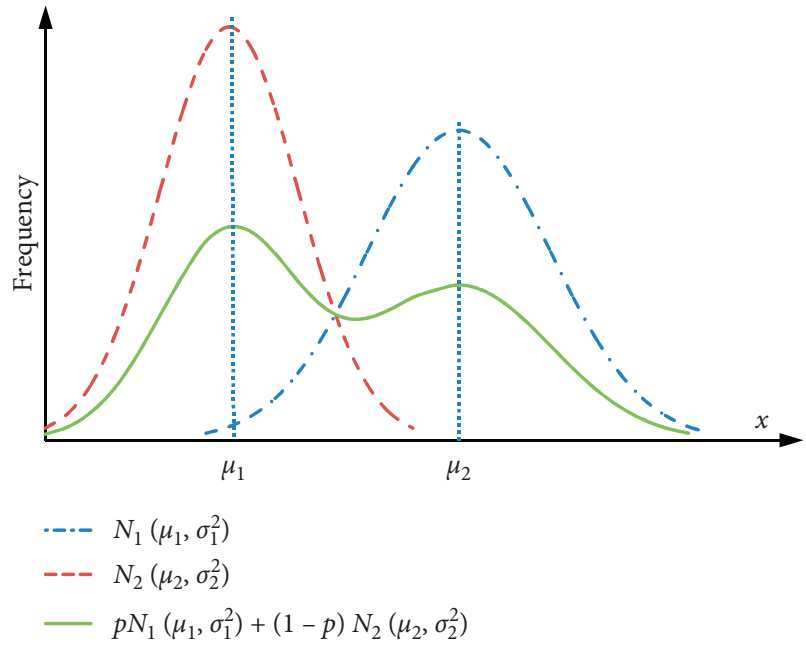

FIGURE 2: Density function diagram of the bi-modal normal distribution.

2.3.2. Association Analysis. The association analysis method is usually used to discover the information from the mass data, and then describe the correlation between a large amount of data. The support and confidence of the association rule are considered, and all the frequent item sets should be found out firstly. The frequency of these item sets should be at least equal to the minimum support frequency set up in advance. Then, the corresponding strong association rule can be generated based on the frequent item sets obtained [12].

Take the Apriori analysis as an example, which is based on the sequential discovery and loop method. Discover the first frequent item set $L_{1}$ from the whole data set, and then discover the second frequent item set $L_{2}$ from the whole data set with $L_{1}$. Repeat this process until no more frequent item sets are found out. The join operation and delete operation are the main two steps of discovering the frequent item set $L_{k}$ with $L_{k-1}$. Firstly, the candidate item set $C_{k}$ can be generated with the join of two item sets of $L_{k-1}$, aiming to discover the frequent item set $L_{k}$. Suppose $l_{1}$ and $l_{2}$ are the item sets of $L_{k-1}, l_{i}[j]$ is the $j$ th term of $l_{i}$, and $l_{i}[k-2]$ is the second last term of $l_{i}$. Suppose the join operation of $L_{k-1}$ is $L_{k-1} \oplus L_{k-1}$, which means the first $(k-2)$ item sets of $l_{1}$ and $l_{2}$ are similar. $\quad\left({ }_{1}[1]=l_{2}[1]\right) \wedge \cdots \wedge\left(l_{1}[k-2]=l_{2}[k-2]\right) \wedge$ $\left(l_{1}[k-1]<l_{2}[k-1]\right)$, and then $l_{1}$ and $l_{2}$ of $L_{k-1}$ can be connected. Secondly, $L_{k} \subseteq C_{k}$, while not all the item sets of $C_{k}$ are the frequent item sets. To reduce the workload of $C_{k}$, an infrequent item set $(k-1)$ cannot be a subset of the frequent item set $(k)$.

Compared with the association analysis method, the traditional correlation analysis method is more widely used to describe the interdependence and closeness of some 
objective things. The statistical indicators are usually implemented, such as the correlation diagram and the correlation coefficient. The correlation diagram is commonly known as the scatter plot, which can be, respectively, expressed in the rectangular coordinate system's $x$-axis and $y$-axis with two variables (Figure 3). Moreover, the correlation coefficient can also be divided into the Pearson correlation coefficient, the Spearman correlation coefficient, and the Kendall correlation coefficient, where the Pearson correlation coefficient has a simple calculation process and can be used in the following calculation and analysis.

2.3.3. Time-Series Analysis. The bridge SHM data are typical time-series data, ranging from 00:00 to 24:00 in a day, and keep repeating every week, every month, and even every year. With the time-series analysis method, a model can be set up to predict the structural response and the structural performance. Moreover, the traffic load can also be predicted with some assumptions of the bridge.

Take the Holt-Winters method as an example, which does not have to store a lot of historical data [25]. The HoltWinters method is often used for nonlinear short-term prediction, which only needs the current actual value and a single smoothing exponent value. With a reasonable smoothing coefficient, the simple prediction of the next period can then be realized. The prediction model is shown as

$$
\tilde{y}_{t+T}=a_{t}+b_{t} \times T+c_{t} \times T^{2},
$$

where $\tilde{y}_{t+T}$ is the prediction value of period $(t+T)$ and $a_{t}, b_{t}$, and $c_{t}$ are different model parameters: $a_{t}=3 s_{t}^{(1)}-3 s_{t}^{(2)}+s_{t}^{(3)}$, $c_{t}=\left(a^{2} / 2(1-a)^{2}\right)\left(s_{t}^{(1)}-2 s_{t}^{(2)}+s_{t}^{(3)}\right), \quad$ and $\quad b_{t}=(a / 2(1-$ $\left.a)^{2}\right) \times\left[(6-5 a) s_{t}^{(1)}-2(5-4 a) s_{t}^{(2)}+(4-3 a) s_{t}^{(3)}\right]$, where $s_{t}^{(1)}$, $s_{t}^{(2)}$, and $s_{t}^{(3)}$ are the first, second, and third smoothing exponents: $\quad s_{t}^{(1)}=a \times y_{t}+(1-a) s_{t-1}^{(1)}, \quad s_{t}^{(2)}=a \times s_{t}^{(1)}+(1-$ a) $s_{t-1}^{(2)}$, and $s_{t}^{(3)}=a \times s_{t}^{(2)}+(1-a) s_{t-1}^{(3)}$.

Additionally, the error analysis is usually used to measure the accuracy of time-series prediction using certain measures, such as the mean error (ME), the mean absolute error (MAE), and the root mean square error (RMSE). A smaller prediction error often means a larger prediction accuracy, and the expressions of different error parameters are shown as

$$
\begin{aligned}
\mathrm{ME} & =\frac{1}{n} \sum_{i=1}^{n}\left(Y_{i}-\widehat{Y}_{i}\right), \\
\mathrm{MAE} & =\frac{1}{n} \sum_{i=1}^{n}\left|Y_{i}-\widehat{Y}_{i}\right|, \\
\mathrm{RMSE} & =\sqrt{\frac{1}{n} \sum_{i=1}^{n}\left(Y_{i}-\widehat{Y}_{i}\right)^{2} .}
\end{aligned}
$$

\section{Predictive Analytics of Structural Response and Deterioration Extent for an Urban CFST Truss Girder Bridge}

3.1. Bridge Overview. The CFST truss girder bridge is located in the central part of China (opened to traffic in 1995). The span arrangement is $(122+62+62+122) \mathrm{m}$. The bridge deck arrangement is $(14.4+14.4) \mathrm{m}$. The main truss beam is designed with the steel tube concrete. The longitudinal beam and crossbeam of the bridge deck are designed with an open steel box. The crossbeam, vertical bar, and sway bar are designed with a steel tube.

In December 2010, some large cracks of the open steel box beam at the bridge pier were discovered, and then the bridge was temporarily closed. The side view of the CFST truss girder bridge is shown in Figure 4. The structural discrete diagram is shown in Figure 5. The static and dynamic load tests were carried out after the appearance inspection. According to the load tests, the stiffness and strength of the longitudinal beam and crossbeam cannot meet the service requirement. Some limit measures were then implemented, and the SHM system was also designed (Table 1 and Figure 6).

3.2. Structural Response Analysis and Prediction. Consider the $62 \mathrm{~m}$ span as an example. The structural responses are analyzed in this section. The structural deterioration extent is also analyzed in the next section.

3.2.1. Structural Response Distribution and Time-Effect Analysis. The distribution of different microstrain $(\mu \varepsilon)$, deflection $(\omega)$, and acceleration is shown in Figures 7 and 8 (ta and va are the transverse and vertical acceleration). Additionally, the variation of different microstrain, deflection, and acceleration in a day is shown in Figures 9 and 10 , where the imaginary line represents the mixing of singleor multi-modal normal distribution. Moreover, different distribution formulas are shown in equations (6) (11), in which Tbt and Bdm represent the truss beam top and the bridge deck midspan:

$$
F_{\mu \varepsilon}^{\mathrm{Tbt}}(x)=0.599 \varphi\left(\frac{x-51.493}{19.294}\right)+0.401 \varphi\left(\frac{x-255.906}{33.192}\right),
$$

$$
\begin{aligned}
& F_{\mu \varepsilon}^{\mathrm{Bdm}}(x)=\varphi\left(\frac{x-130.000}{10.000}\right), \\
& F_{w}^{\mathrm{Bdm}}(x)=\varphi\left(\frac{x-7.680}{5.120}\right), \\
& F_{\mathrm{ta}}^{\mathrm{Tbt}}(x)=\varphi\left(\frac{x-0.805}{0.374}\right), \\
& F_{\mathrm{va}}^{\mathrm{Tbt}}(x)=\varphi\left(\frac{x-0.947}{0.440}\right),
\end{aligned}
$$




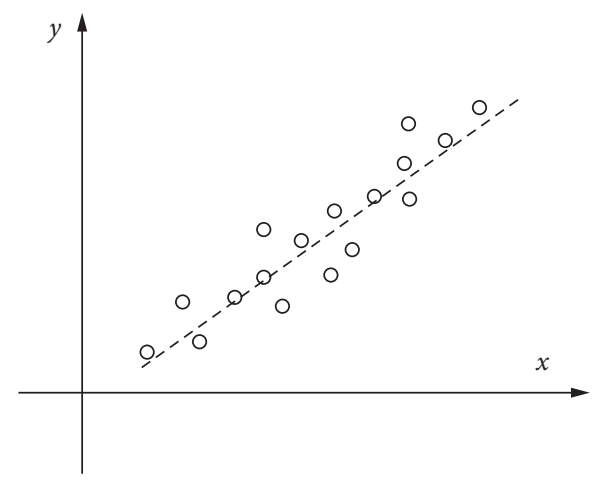

(a)

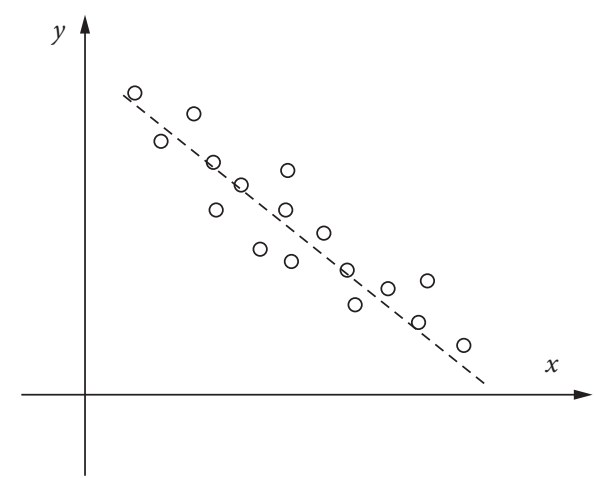

(b)

Figure 3: Diagrams of the positive correlation (a) and negative correlation (b).

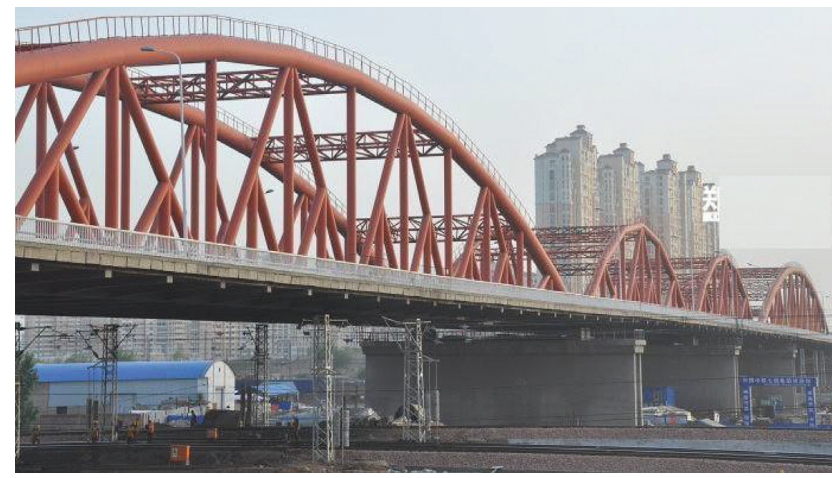

FIgURE 4: Side view of the bridge.

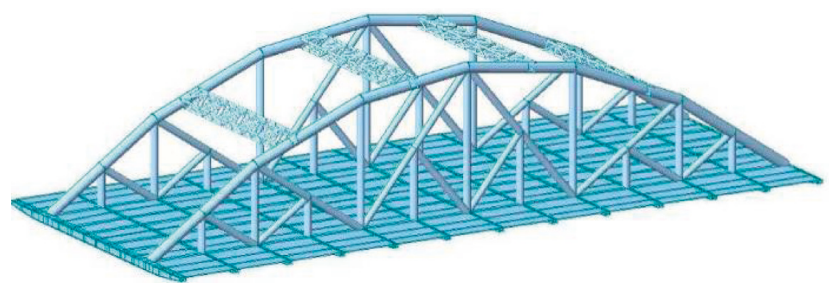

FIgURE 5: Structural discrete diagram of the bridge.

TABLE 1: Sensor arrangement of the bridge SHM system.

\begin{tabular}{lccc}
\hline Sensor type & Location & Number & Remarks \\
\hline Deflection & 1,3 & $1+1$ & Bridge deck center \\
\hline \multirow{2}{*}{ Microstrain } & 1,3 & $2+2$ & Bridge deck center \\
& 2,5 & $1+1$ & Truss beam top \\
\hline \multirow{4}{*}{ Acceleration } & 1,3 & $1+1$ & Bridge deck center \\
& 2,5 & $2+2$ & Truss beam top \\
& 4,6 & $2+2$ & $l / 4$ and $3 l / 4$ of the truss beam \\
\hline \multirow{2}{*}{ Temperature } & 3 & 2 & Bridge deck center \\
& 5 & 2 & Truss beam top \\
\hline
\end{tabular}

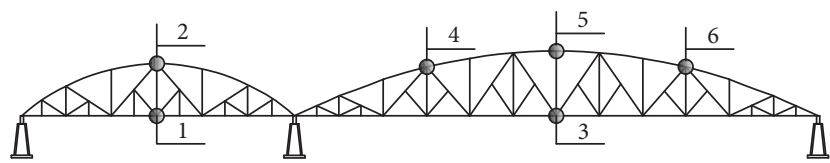

FIGURE 6: Measurement points of the bridge SHM system.

$$
F_{\mathrm{va}}^{\mathrm{Bdm}}(x)=0.512 \varphi\left(\frac{x-0.141}{0.022}\right)+0.488 \varphi\left(\frac{x-0.115}{0.053}\right) .
$$

According to Figures 7 and 8 and equations (6) (11), the microstrain, deflection, and acceleration almost follow a different distribution. Most of the SHM data concentrate on average area, and then the weak sensibility of the structure subjected to traffic load can be reflected. According to the inspection and test report, it is found to be due to the weak stiffness and strength of the longitudinal beam and crossbeam. The different distribution can also be fitted as the mixing of single- or multi-modal normal distribution function. For example, the microstrain of the truss beam top follows the bi-modal distribution (the average value of part one is 51.493, and the average value of part two is 255.906), while the microstrain of the bridge deck midspan follows the single-modal distribution (the average value is 130).

According to Figures 9 and 10, most of the deflection and microstrain are small, and only some large data are observed. According to the additional video monitoring system, the large data are a result of the individual vehicles or trucks and have a little effect on the overall structural safety. The deflection and microstrain in a day have a different variation tendency, while different accelerations almost follow a similar variation tendency. The SHM data can be divided into part one (from 00:00 to 05:00) and part two (from 05:00 to $24: 00$, especially from 10:00 to 16:00). According to the additional video monitoring system, the former is caused by the high-speed garbage or slag truck during the nighttime and the latter is caused by the bus or private car, of which the latter is significant than the former. The data almost rise gradually from 08:00, and the crests appear at around 11:00 or 15:00. Afterward, the deflection and microstrain decline gradually, and the troughs appear at around 05:00 or 24:00.

3.2.2. Structural Response Prediction. Take the vertical acceleration of the bridge deck midspan from 15 at 00:00 in the morning to 18 at 24:00 in the evening as an example. The periodic effect, filtering, and forecast of the vertical acceleration of the bridge are shown in Figure 11, where the dotted line represents the measurement data and the left red line the method verification. The right blue line and the 


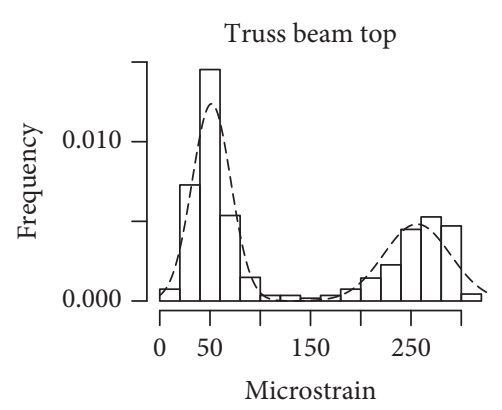

(a)

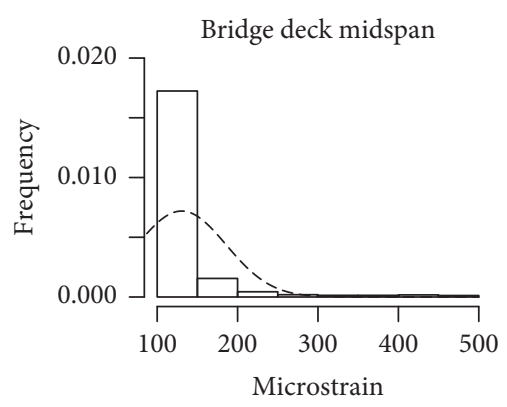

(b)

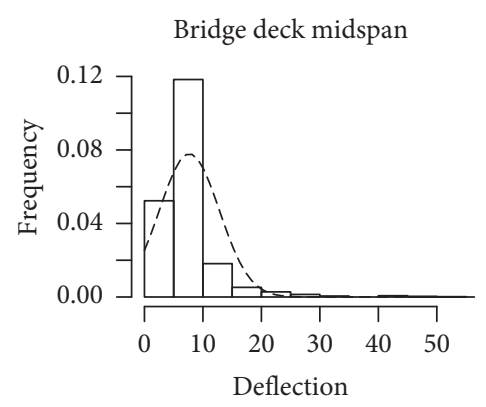

(c)

FIgURE 7: Distribution diagram of microstrain and deflection $(\mathrm{mm})$ of the bridge.

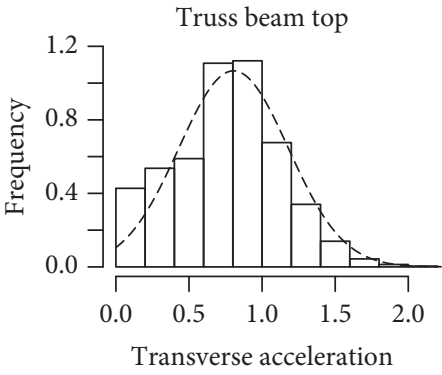

(a)

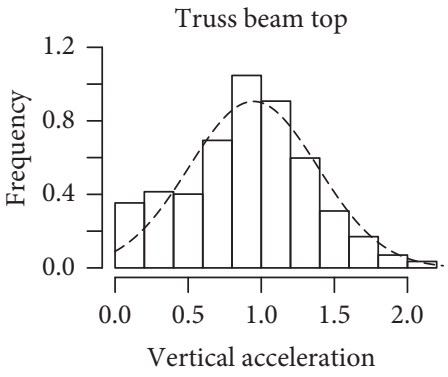

(b)

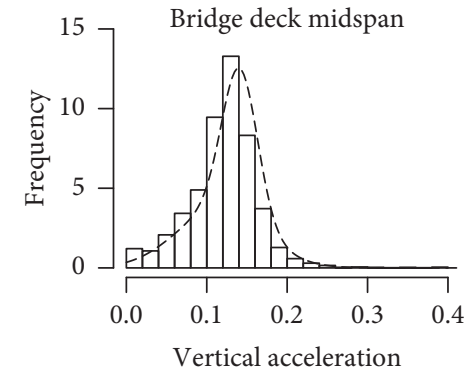

(c)

FIgURE 8: Distribution diagram of acceleration $\left(\mathrm{m} / \mathrm{s}^{2}\right)$ of the bridge.

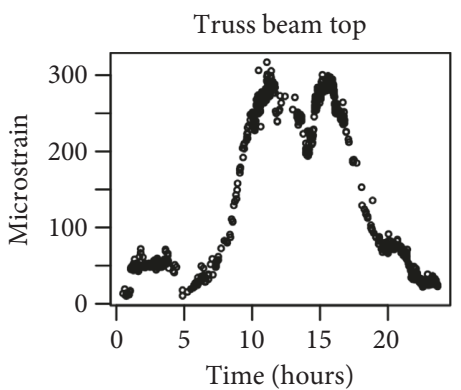

(a)

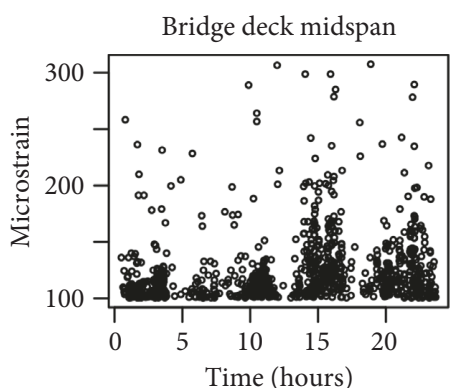

(b)

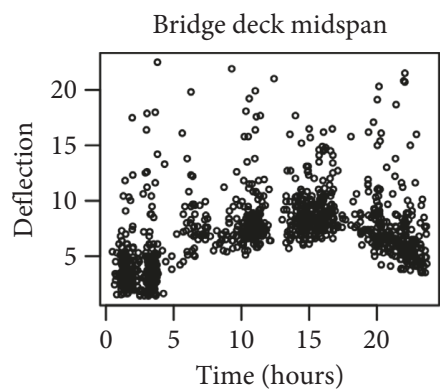

(c)

FIgURE 9: Microstrain and deflection ( $\mathrm{mm}$ ) variation for 24 hours of the bridge.

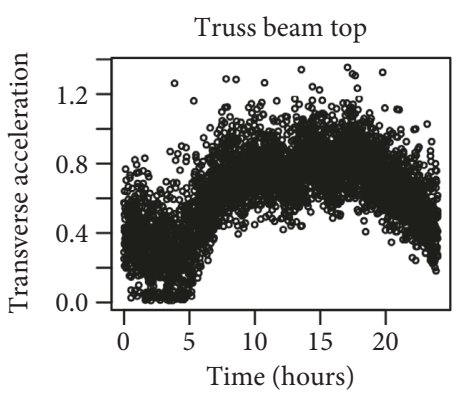

(a)

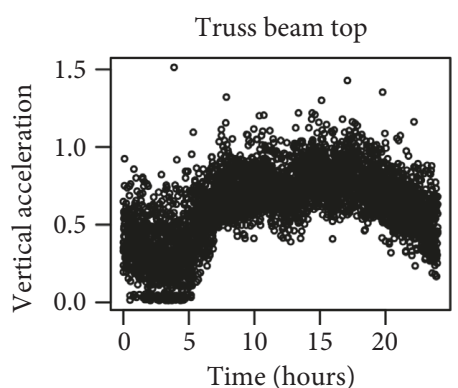

(b)

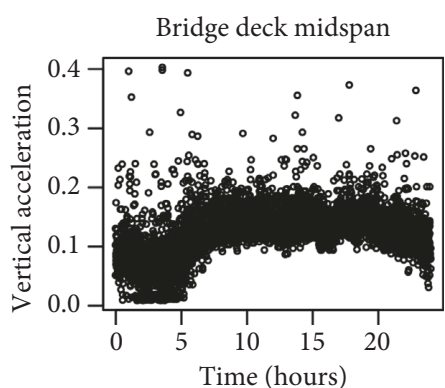

(c)

Figure 10: Acceleration $\left(\mathrm{m} / \mathrm{s}^{2}\right)$ variation for 24 hours of the bridge. 


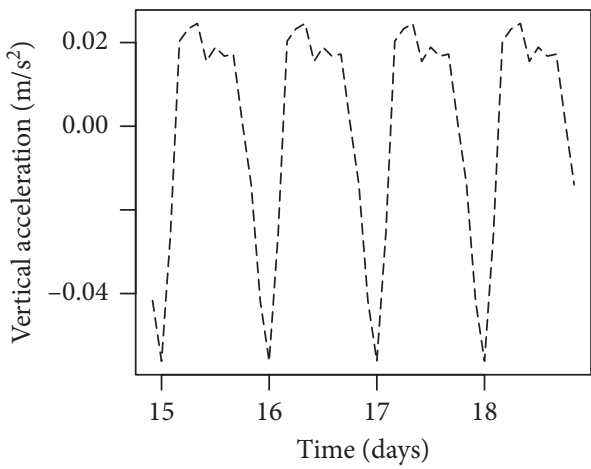

(a)

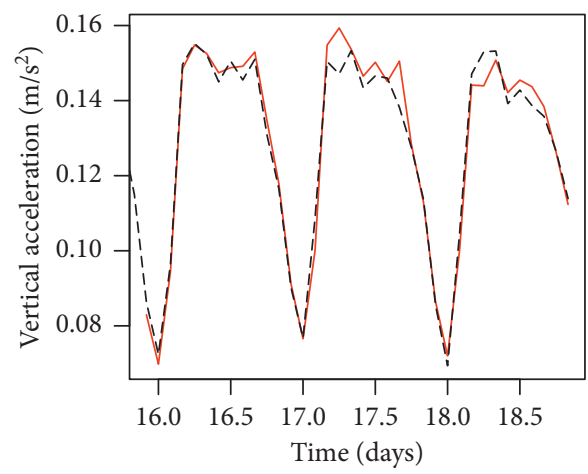

(c)

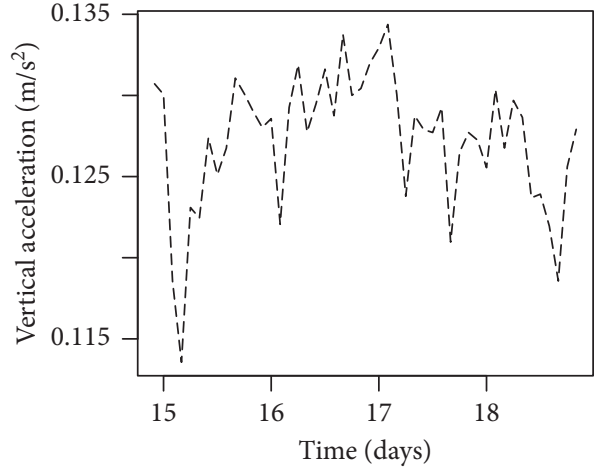

(b)

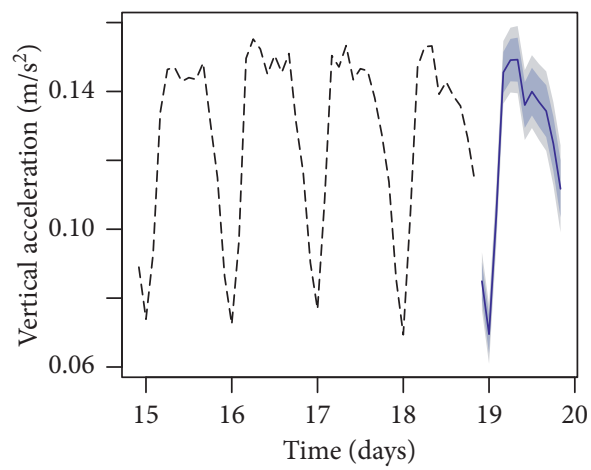

(d)

Figure 11: Periodic effect, filtering, and forecast of the vertical acceleration $\left(\mathrm{m} / \mathrm{s}^{2}\right)$ of the bridge deck midspan. (a) Periodic effect. (b) Effect without period. (c) Holt-Winters filtering. (d) Forecast from Holt-Winters filtering.

shaded area show the prediction result with confidence intervals. Moreover, the prediction error is still fine, and different prediction error parameters are also shown in Table 2.

According to Figure 11, the left dotted line of the periodic effect is significantly smaller than the right dotted line of the random effect of different days. This reflects that the periodic effect is smaller than the random effect of different days. For the periodic effect, the dotted line has a significant decrement in the afternoon of different days, and it also has a significant increment in the morning of different days. The maximum value of the dotted line appears around noon. This also reflects the variation trend of the vertical acceleration with the periodic effect. According to the additional video monitoring system, this is due to different traffic volume of different hour stages. Moreover, the left red line almost coincides with the dotted line. This reflects that the Holt-Winters method can match the measurement data to a great extent. This can also be verified by a small prediction error in Table 2. The right blue line shows the vertical acceleration of the next few short periods, and the confidence interval is also shown in the shaded area. Thus, the vertical acceleration of the next few short periods can be further predicted.

\subsection{Structural Deterioration Extent Analysis and Prediction}

3.3.1. Structural Deterioration Extent Analysis. Take the microstrain and deflection of the bridge deck midspan as an
TABle 2: Prediction error of structural response (\%).

\begin{tabular}{lccc}
\hline Prediction type & ME & RMSE & MAE \\
\hline Vertical acceleration & 0.001 & 0.004 & 0.003
\end{tabular}

example, and the diagram of microstrain and deflection correlation is shown in Figure 12. Moreover, the diagrams of different acceleration and microstrain are shown in Figure 13, where TB.t.ta, TB.t.va, and BD.m.va represent the transverse acceleration of the truss beam top, the vertical acceleration of the truss beam top, and the vertical acceleration of the bridge deck midspan.

According to Figure 12, most of the microstrain and deflection data of the bridge deck midspan are scattered. Therefore, there is a significant weak correlation for the microstrain and deflection of the bridge deck midspan. This reflects that the bridge structure has a complex mechanical behavior and significant deterioration after decades of years of service. Some reinforcement measures can still be further implemented so as to improve the correlation of the deflection and microstrain of the bridge deck midspan.

According to Figure 13, most of the transverse and vertical acceleration data of the truss beam top follow the linearity change trend. Therefore, there is still a good transverse and vertical acceleration correlation for the truss beam top, while the vertical acceleration data of the truss beam top and bridge deck midspan are a little scattered, 


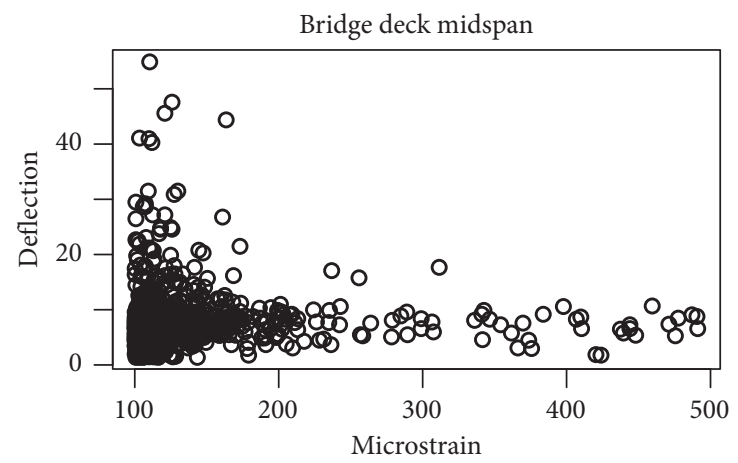

FIgURE 12: Correlation diagram of microstrain and deflection $(\mathrm{mm})$ of the bridge.

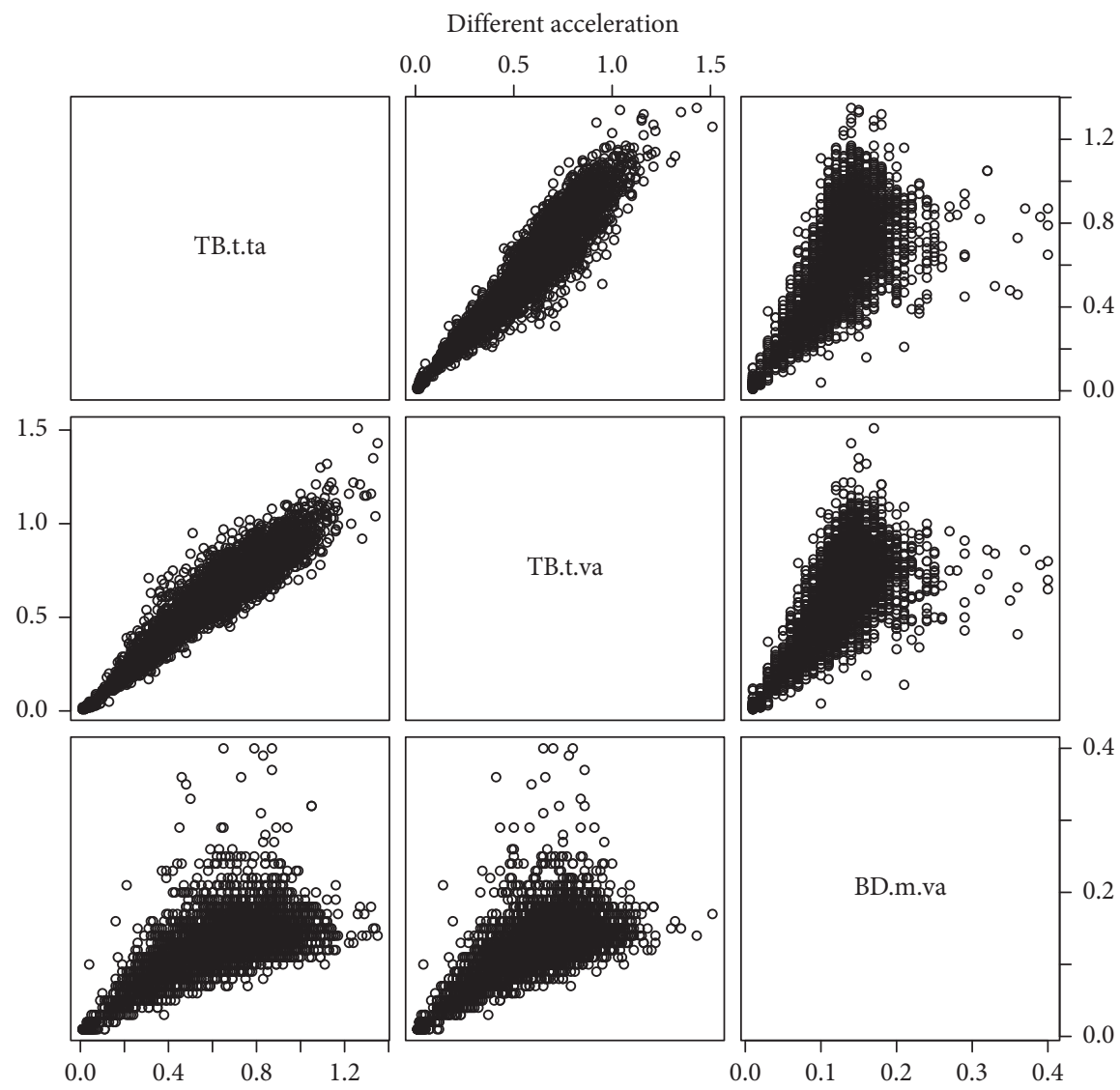

FIGURE 13: Correlation diagram of different acceleration $\left(\mathrm{m} / \mathrm{s}^{2}\right)$ of the bridge.

which means a little weak vertical acceleration correlation. This reflects that the vibration behavior of the truss beam still works fine, while the vibration behavior of the bridge deck midspan still needs some further improvement.

3.3.2. Structural Deterioration Extent Prediction. Take the transverse and vertical acceleration correlation of the truss beam top from 10 at 00:00 to 13 at 24:00 as an example. The periodic effect, filtering, and forecast of the transverse and vertical acceleration correlation are shown in Figure 14, where the dotted line represents the measurement data and the left red line the method verification. The right blue line and the shaded area show the prediction result with confidence intervals. The prediction also has a small error, and different prediction error parameters are also shown in Table 3.

According to Figure 14, the left dotted line with the periodic effect is significantly smaller than the right dotted line with the random effect of different days. This reflects that the periodic effect is significantly smaller than the random effect of different days. For the periodic effect, the dotted line has a significant decrement in the morning of different days, and it also has a significant increment in the afternoon of different days. The minimum value of the dotted line appears around noon. This also reflects the 


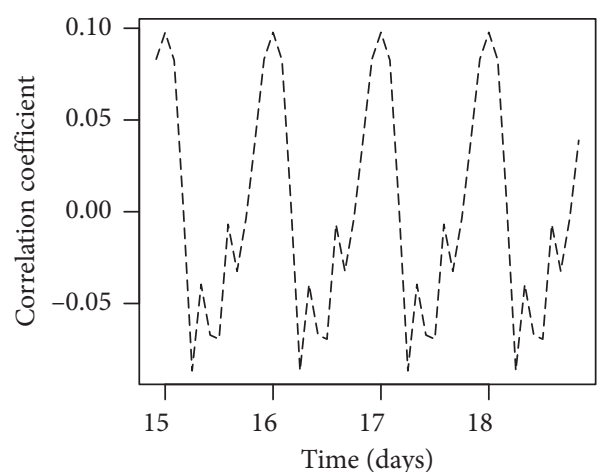

(a)

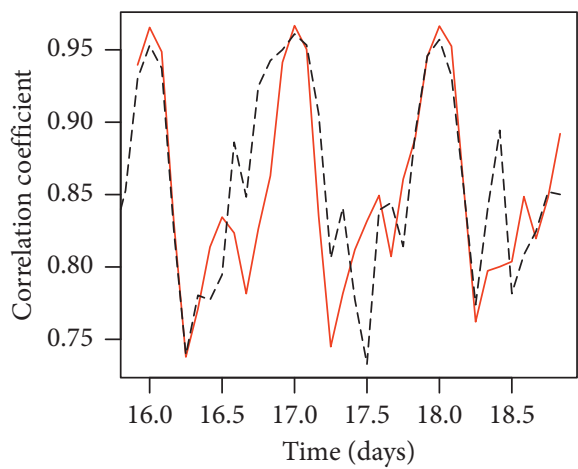

(c)

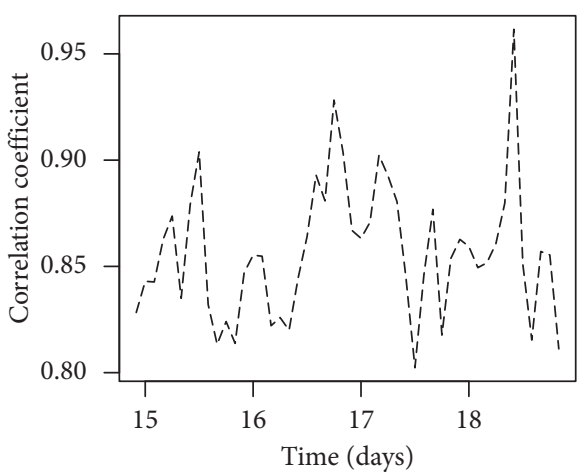

(b)

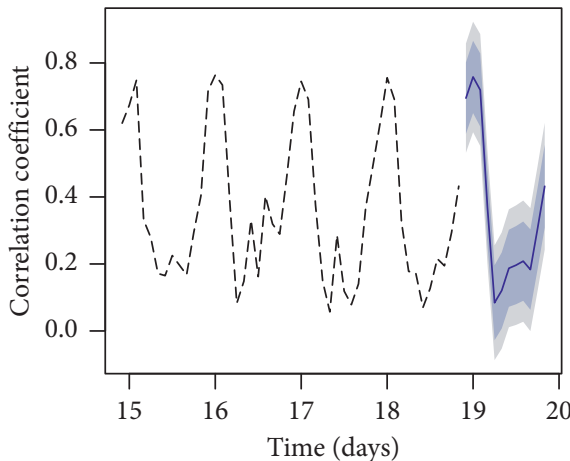

(d)

Figure 14: Periodic effect, filtering, and forecast of transverse and vertical acceleration correlation of the truss beam top. (a) Periodic effect. (b) Effect without period. (c) Holt-Winters filtering. (d) Forecast from Holt-Winters filtering.

Table 3: Prediction error of structural deterioration extent (\%).

\begin{tabular}{lccc}
\hline Prediction type & ME & RMSE & MAE \\
\hline $\begin{array}{l}\text { Acceleration correlation (transverse and } \\
\text { vertical) }\end{array}$ & 0.008 & 0.044 & 0.032 \\
\hline
\end{tabular}

transverse and vertical acceleration correlation's variation trend with the periodic effect. According to the additional video monitoring system, this is due to different traffic volume of different hour stages. Moreover, the left red line coincides with the dotted line to a certain extent. This reflects that the Holt-Winters method can match the measurement data to a certain extent. This can also be verified by a small prediction error in Table 3. The right blue line shows the transverse and vertical acceleration correlation of the next short periods, and the confidence interval is also shown in the shaded area. Thus, the transverse and vertical acceleration correlation of the next few short periods can be further predicted.

\section{Discussion}

This study focuses on the structural performance analysis and prediction of an in-service bridge. The dynamic problems in bridge SHM are pointed out firstly. A detailed analysis about the characteristics of bridge SHM data is then carried out, and then three targeted predictive analytic methods are proposed. (i) The essential dynamic problem of the traffic load, the bridge structure, and the structural response in bridge SHM is pointed out firstly, including the forward and inverse problems. The typical characteristics of bridge SHM data are then analyzed, such as the variation along with different traffic loads and different time periods. This section is important for the whole study, which can also be accepted widely for bridge SHM.

(ii) With the distribution function, the structural responses are clearly presented in the multi-modal distribution diagram. The sensibility of the bridge structure subjected to traffic load can then be reflected. Moreover, the variation trend of traffic volume of different time periods in a day is also clearly presented, such as the busy period and maximum phase of the traffic volume. This section provides a reference for the overall structural safety. The variation trend of traffic volume can also be used for decision-making by the maintenance and traffic management department.

(iii) The association analysis method is employed to reflect the mapping relationship of structural parameters (stress, displacement, etc.). As there will be a weak correlation distribution of different parameters after a long periodical service, the structural distribution performance and deterioration extent can be reflected. This section provides 
a reference for the mechanical performance and deterioration extent, and then can be used for decision-making by the maintenance department.

(iv) With the time-series analysis method, the structural response and its correlation coefficient are predicted to a certain extent. The division of the periodic effect and random effect is carried out firstly. The prediction error is also employed to check the prediction result. Then, the prediction of the next few periods can be realized with confidence interval. This section provides a short-period prediction of the structural response and deterioration extent, and then can be used for decision-making by the maintenance and traffic management department.

\section{Conclusion}

The CFST truss girder bridge still has a good overall structural safety, while the bridge deck midspan needs some further reinforcement so as to improve the stiffness and strength of the longitudinal beam and crossbeam. Also, complex mechanical behavior and significant deterioration for the bridge deck midspan are observed, whereas the truss beam still has a good vibration performance. Moreover, the proposed SHM data mining methods can also be used for the analysis and prediction of structural performance for other kinds of bridges.

It is recommended to try to avoid driving from around 11:00 or 15:00, as well as around 00:00 to 05:00. Leaving home early at 07:00 and returning at 18:00 is also advised. Some traffic restriction measures can be implemented at around 11:00 or 15:00. The speed limit of the garbage or slag truck can also be implemented at around 00:00 to 5:00.

\section{Data Availability}

The primary data used to support the findings of this study are available from the corresponding author upon request (e-mail: jqw.sinx123@163.com).

\section{Conflicts of Interest}

The authors declare that they have no conflicts of interest.

\section{Acknowledgments}

The support from the Henan Transportation Research Institute Co., Ltd, is gratefully acknowledged, which once carried out several inspections, load tests, and SHM for the bridge case study in this paper.

\section{References}

[1] D. Pines and A. E. Aktan, "Status of structural health monitoring of long-span bridges in the United States," Progress in Structural Engineering and Materials, vol. 4, no. 4, pp. 372380, 2002.

[2] S. Bas, N. M. Apaydin, A. Ilki, and F. N. Catbas, "Structural health monitoring system of the long-span bridges in Turkey,"
Structure and Infrastructure Engineering, vol. 14, no. 4, pp. 425-444, 2017.

[3] Z. Sun, Z. Zou, and Y. Zhang, "Utilization of structural health monitoring in long-span bridges: case studies," Structural Control and Health Monitoring, vol. 24, no. 10, article e1979, 2017.

[4] A. Basheer, K. Huma, B. Baidar, M. Aftab, and R. Jonathan, "Some observations on BWIM data collected in Manitoba," in Proceedings of the 10th International Conference on Short and Medium Span Bridges, Quebec, Canada, July-August 2018.

[5] M. Richardson, "Detection of damage in structures from changes in their dynamic (modal) properties-a survey (NO. UCRL-15103)," Technical report, Structural Measurement Systems, Inc., 1980.

[6] D. F. Mazurek and J. T. DeWolf, "Experimental study of bridge monitoring technique," Journal of Structural Engineering, vol. 116, no. 9, pp. 2532-2549, 1990.

[7] G. Hearn and R. B. Testa, "Modal analysis for damage detection in structures," Journal of Structural Engineering, vol. 117, no. 10, pp. 3042-3063, 1991.

[8] J. E. Mottershead and M. I. Friswell, "Model updating in structural dynamics: a survey," Journal of Sound and Vibration, vol. 167, no. 2, pp. 347-375, 1993.

[9] N. Stubbs, S. Park, C. Sikorsky, and S. Choi, "A global nondestructive damage assessment methodology for civil engineering structures," International Journal of Systems Science, vol. 31, no. 11, pp. 1361-1373, 2000.

[10] Q. Zhang, J. Zhang, W. Duan, and Z. Wu, "Deflection distribution estimation of tied-arch bridges using long-gauge strain measurements," Structural Control and Health Monitoring, vol. 25, no. 3, article e2119, 2018.

[11] J.-X. Mao, H. Wang, D.-M. Feng, T.-Y. Tao, and W.-Z. Zheng, "Investigation of dynamic properties of long-span cablestayed bridges based on one-year monitoring data under normal operating condition," Structural Control and Health Monitoring, vol. 25, no. 5, article e2146, 2018.

[12] D. Cheng, "The application and research of association analysis in traditional Chinese medicine data mining," Master's thesis, Beijing Jiaotong University, Beijing, China, 2006.

[13] K. Fang, "A study on algorithm of classification and cluster based on data mining and realization by $\mathrm{R}$ programe," Master's thesis, Jinan University, Guangzhou, China, 2007.

[14] D. Galar, A. Gustafson, B. Martínez, and L. Berges, "Maintenance decision making based on different types of data fusion," Eksploatacja i Niezawodnosc-Maintenance and Reliability, vol. 14, pp. 135-144, 2012.

[15] M. Gordan, H. A. Razak, Z. Ismail, and K. Ghaedi, "Recent developments in damage identification of structures using data mining," Latin American Journal of Solids and Structures, vol. 14, no. 13, pp. 2373-2401, 2017.

[16] L. Li, J. Wang, H. Leung, and C. Jiang, "Assessment of catastrophic risk using Bayesian network constructed from domain knowledge and spatial data," Risk Analysis, vol. 30, no. 7, pp. 1157-1175, 2010.

[17] A. Dutta, "Bridge strain data analysis using density based clustering technique," in Proceedings of the 2015 IEEE International Conference on Research in Computational Intelligence and Communication Networks (ICRCICN), Kolkata, India, November 2015.

[18] S. Lim, S. Chung, S. Chi, and J. Song, "A framework for developing an estimation model of damages on bridge elements using big data analytics," in Proceedings of the 33rd ISARC, Auburn, AL, USA, 2016. 
[19] Y. Q. Ni, X. G. Hua, K. Q. Fan, and J. M. Ko, "Correlating modal properties with temperature using long-term monitoring data and support vector machine technique," Engineering Structures, vol. 27, no. 12, pp. 1762-1773, 2005.

[20] H. Dong, "Data mining method for bridge health monitoring data," Master's thesis, Chongqing University, Chongqing, China, 2006.

[21] S. Tesfamariam and Z. Liu, "Earthquake induced damage classification for reinforced concrete buildings," Structural Safety, vol. 32, no. 2, pp. 154-164, 2010.

[22] Z. Liu and S. Tesfamariam, "Prediction of lateral spread displacement: data-driven approaches," Bulletin of Earthquake Engineering, vol. 10, no. 5, pp. 1431-1454, 2012.

[23] N. Gulgec, G. Shahidi, T. Matarazzo, and S. Pakzad, "Current challenges with bigdata analytics in structural health monitoring," in Proceedings of the 35th IMAC, Garden Grove, CA, USA, 2017.

[24] D. Hu and Y. Zhou, "Study on the design vehicle load model and technical parameters of highway bridge in china (no. XBKYZT-03)," Technical report, Changan University, Xi'an, China, 2011.

[25] X. Yan, C. He, and Y. Huang, "The application of HoltWinters method in building accident prediction," Statistics and Decision, vol. 4, pp. 72-73, 2015. 


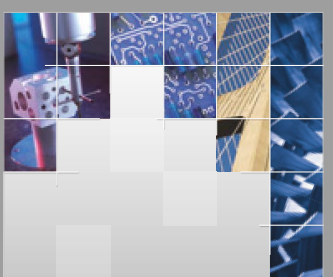

\section{Enfincering}
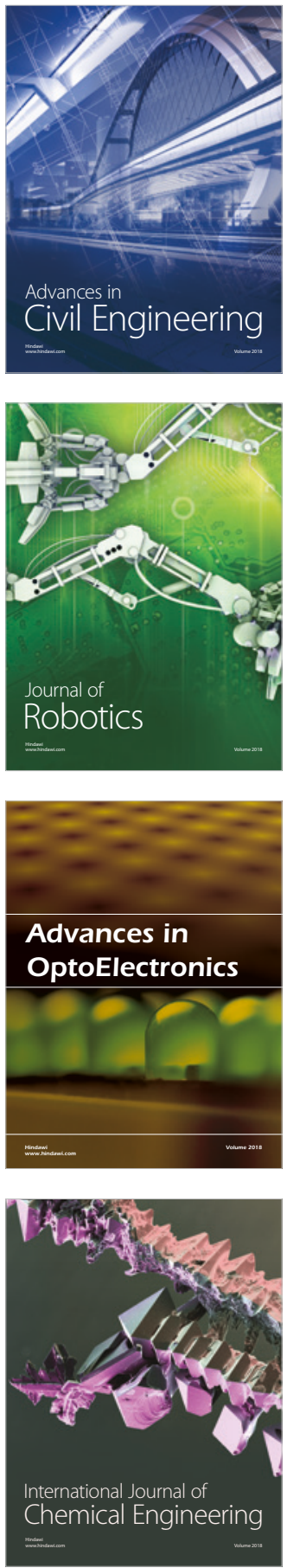

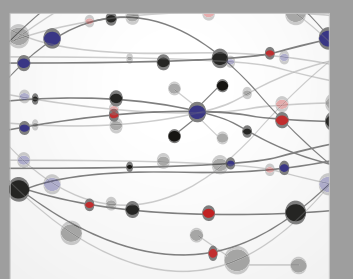

\section{Rotating \\ Machinery}

The Scientific World Journal

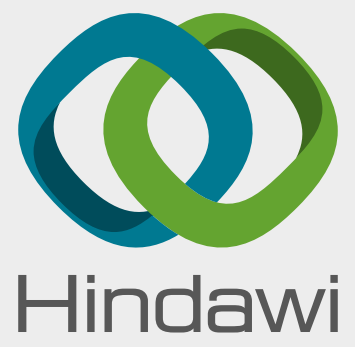

Submit your manuscripts at

www.hindawi.com
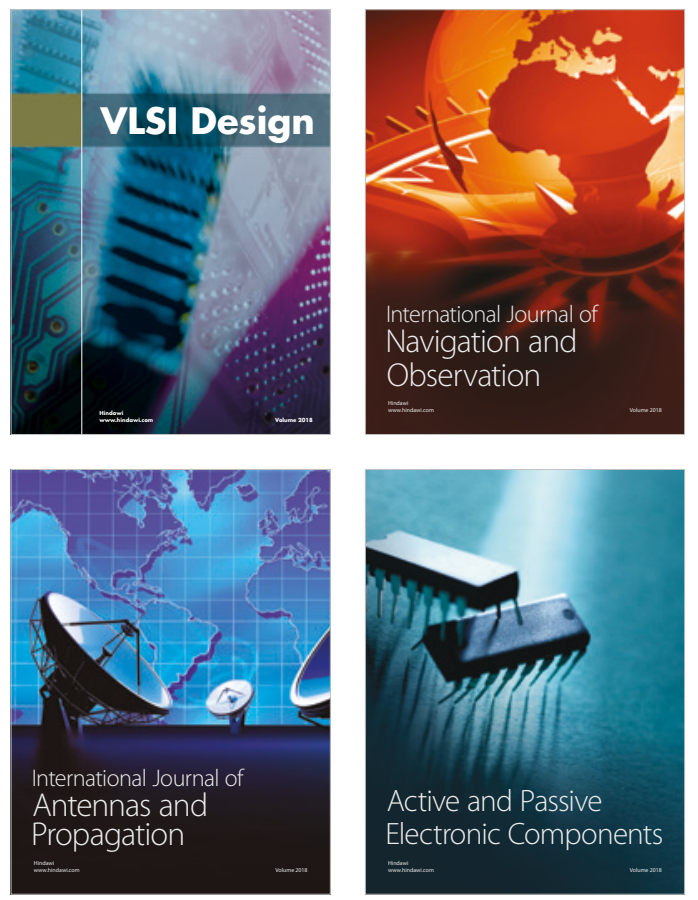
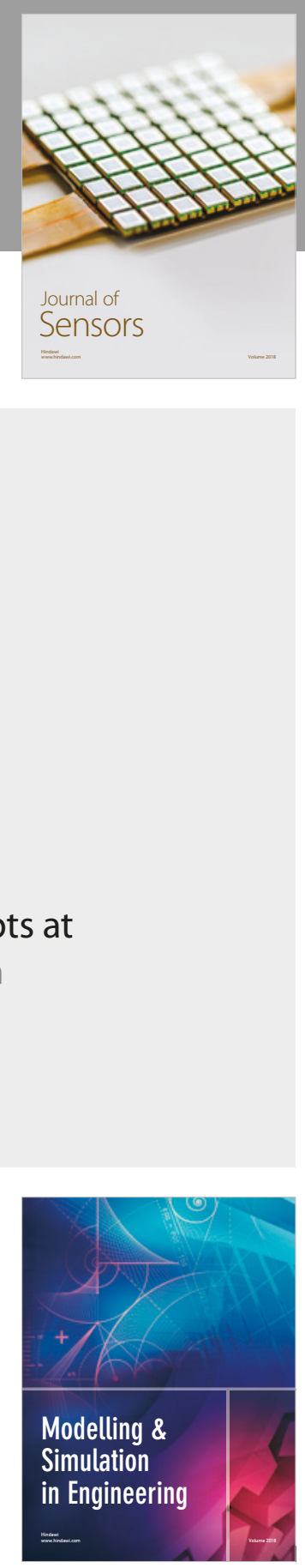

\section{Advances \\ Multimedia}
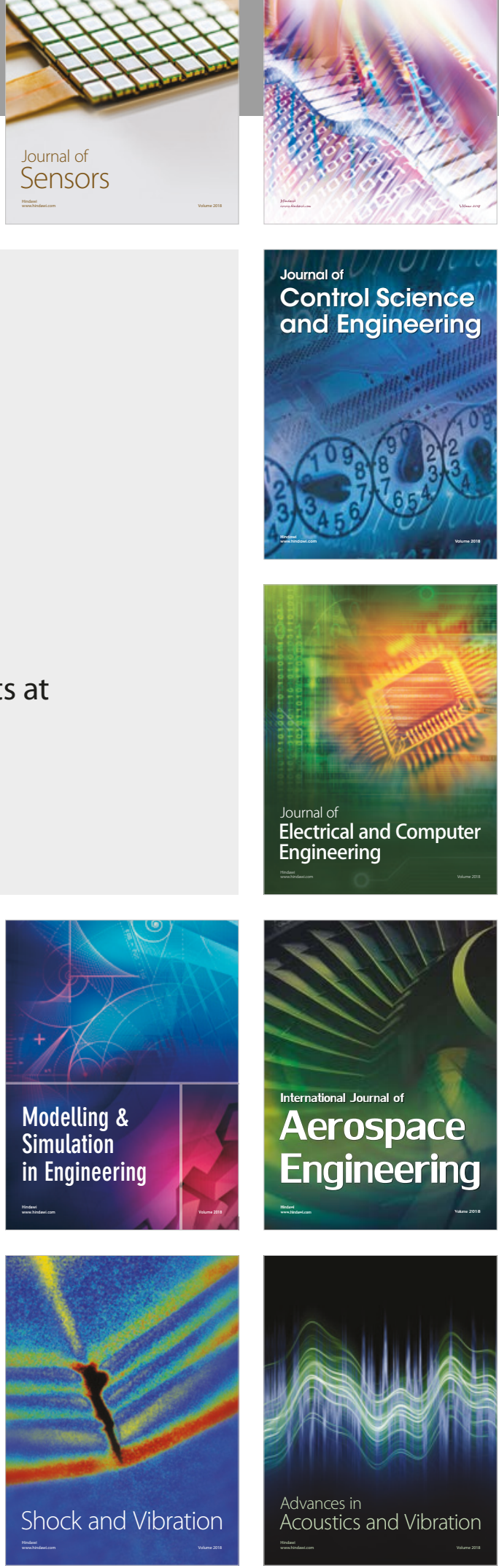